\title{
MEETINGS/ CONFERENCES/ SYMPOSIA/ WORKSHOP/ SEMINARS
}

November 21-24, 2001

Workshop on Advancing Bagasse-Based Cogeneration

Hotel Hyatt Regency, New Delhi

Contact : Mr. CVJ Varma

Secretary General, Council of Power Utilities

A-2/158, Janakpuri, New Delhi - 110058 , India

Tel. : 91-11-5618472; Fax : 91-11-5611652

e-mail : cvj@vsnl.com

November 27-28, 2001

10th International Sugar Organization Seminar

Cabot Hall, London, UK. "Movers and Shakers -Their Impact on the World Sweereners Market."

Contact : (44) (0)20 75131144

Fax : (44)(0)20 75131146

e-mail : exdir@ isosugar.org.

February 16-18, 2002

International Symposium on Food, Nutrition and Economic Security through Diversification in Sugarcane Production and Processing Systems, Lucknow, UP, India Contact : Dr. Menhi Lal

Organizing Secretary, International Symposium, Indian Institute of Sugarcane Research, Lucknow - 226002 , India Tel. : 091-522-480735, 480736; Fax : 91-522-480738

e-mail : asti_iisr@yahoo.com.in asti_iisr@rediffmail.com
March 10-13, 2002

Conference of Sugar Processing Research

Wyndham Hotel, New Orleans, LA.

Contact :

Phone : 504-286-4329,

Fax : 504-282-5387, e-mail : spri@srrc.ars.usda.gov.

May 16-17, 2002

First Biannual World Conference on Recent Developments in Sugar Technologies

Delray Beach, FL.

Sponsored by : Dr. Chou Technologies

Contact : e-mail : DrChouUSA@aol.com

January 16-21, 2005

XXV ISSCT Congress

Contact : Secretary/Treasurer

Mauricio Cobarrus, Vice-President

Cengicana, 6a, Calle 6-38

Zona-9, Edificio Tivoli

Officiana 6, Sotano

Guatemala City

e-mail : cengican@concyt.gob.gt centro@cengicana.org

Tel. : (502)331-3068

Fax : (502)334-0635 